\title{
Insulin induces expression of the hepatic vaspin gene
}

\author{
Daisuke Aibara, Kohei Matsuo, Shigeru Yamano and Kimihiko Matsusue
}

Faculty of Pharmaceutical Science, Fukuoka University, Fukuoka 814-0180, Japan

\begin{abstract}
Visceral adipose tissue-derived serine protease inhibitor (vaspin), initially identified in the visceral adipose tissue, is an adipokine that improves endoplasmic reticulum stress in obesity or insulin sensitivity and glucose tolerance. However, the transcriptional regulation of the hepatic vaspin gene remains elusive. We have previously shown that CCAAT-enhancerbinding protein $\alpha$, a transcription factor of the basic leucine zipper class, positively regulates the vaspin gene. The present study aimed to investigate the nutritional or hormonal regulators of vaspin expression in the liver. For the fasting and refeeding study, mice in the fasting group were subjected to fasting for $24 \mathrm{~h}$ and then sacrificed. Mice in the refeeding group were subjected to fasting for $24 \mathrm{~h}$ and then refed with a $50 \%(\mathrm{w} / \mathrm{w})$ sucrose/MF diet for further $24 \mathrm{~h}$ and then sacrificed. For the streptozotocin (STZ) study, STZ $(50 \mathrm{mg} / \mathrm{kg}$ ) was intraperitoneally injected into C57BL/6JJc1 mice for $5 \mathrm{~d}$. Hepatic vaspin was repressed due to fasting for $24 \mathrm{~h}$ and was induced upon refeeding with a high-sucrose diet. In studies on liver-specific $\mathrm{C} /$ $\mathrm{EBP} \alpha$-deficient mice, $\mathrm{C} / \mathrm{EBP} \alpha$ was not involved in the induction of hepatic vaspin upon refeeding. In addition, the depletion of insulin by streptozotocin treatment markedly decreased hepatic vaspin expression. Finally, fasting-repressed vaspin expression in the liver was significantly increased by direct injection of insulin into fasting mice. In conclusion, our results suggest that insulin is a positive regulator of hepatic vaspin expression.
\end{abstract}

Key words: Insulin, Vaspin, CCAAT-enhancer-binding proteins, Hepatokine

VISCERAL ADIPOSE TISSUE-DERIVED SERINE PROTEASE INHIBITOR (VASPIN) was initially identified in Otsuka Long-Evans Tokushima Fatty (OLETF) rats, an animal model for abdominal obesity with type 2 diabetes; vaspin belongs to the serpin A12 serine protease inhibitor family [1] and is an adipokine that is able to improve insulin sensitivity and glucose tolerance, as demonstrated by administration of the recombinant human vaspin protein to mice with dietinduced obesity [1]. It has been found that vaspin interacts with the 78-kDa glucose-regulated protein (GRP78)/ murine tumor cell DnaJ-like protein 1 (MTJ-1) complex in mouse liver, resulting in the activation of intracellular Akt and AMPK signaling, leading to improvement of insulin sensitivity in mice fed a high-fat diet [2]. In addition, vaspin acts as a ligand for the cell-surface GRP78/ voltage-dependent anion channel complex in endothelial cells, promotes proliferation, inhibits apoptosis, and protects against diabetes mellitus-associated vascular injury [3].

Recent studies on vaspin have focused on its physio-

Submitted Jul. 1, 2019; Accepted Aug. 2, 2019 as EJ19-0276 Released online in J-STAGE as advance publication Sep. 4, 2019 Correspondence to: Kimihiko Matsusue, Faculty of Pharmaceutical Science, Fukuoka University, 8-19-1 Nanakuma, Jonan-ku, Fukuoka 814-0180, Japan.

E-mail: matsusuk@fukuoka-u.ac.jp logical functions; however, the mechanism underlying the regulation of the vaspin gene remains unknown. Interestingly, vaspin expression was restricted to visceral fat in OLETF rats but not observed in major tissues including the liver. In addition, vaspin mRNA level was higher in OLETF rats than in non-diabetic control rats [1]. Further, vaspin expression was observed in humans $[4,5]$ or mice [6] in several tissues such as adipose tissue, liver, skeletal muscle, pancreas, and skin, with the highest production being observed in the liver or skin. Recently, we demonstrated that the vaspin gene in the liver is positively regulated by CCAAT-enhancer-binding protein $\alpha(\mathrm{C} / \mathrm{EBP} \alpha)$ through $\mathrm{C} / \mathrm{EBP} \alpha$ binding sites located in the vaspin promoter (Submitted). It is known that insulin not only rapidly dephosphorylates the $\mathrm{C} / \mathrm{EBP} \alpha$ protein and reduces its mRNA expression, but also induces the expression of $\mathrm{C} / \mathrm{EBP} \beta$ and $\mathrm{C} / \mathrm{EBP} \delta$ genes $[7,8]$. Therefore, we aimed to investigate the mechanism underlying hormonal or nutritional regulation of hepatic vaspin expression through $\mathrm{C} / \mathrm{EBPs}$.

\section{Materials and Methods}

\section{Animal studies}

All animal protocols and studies were performed according to guidelines of the Center for Experimental Animals at Fukuoka University, Japan. Liver-specific 
$\mathrm{CEBP} \alpha$ knockout mice $(\mathrm{C} / \mathrm{EBP} \alpha \mathrm{LKO})$ were generated by breeding the $c / e b p$-floxed mice with mice expressing Cre recombinase under the control of the albumin promoter, as described previously [9, 10]. The tissue distribution of vaspin mRNA was examined using each tissue from C57BL/6JJc1 mice (male, 12 weeks). For the fasting and refeeding study, C57BL/6JJc1 mice (male, 10 weeks) were fed a regular chow diet (MF, Oriental Yeast, Tokyo, Japan) ad libitum until the experimental treatment commenced. Mice in the fasting group $(n=3)$ were subjected to fasting for $24 \mathrm{~h}$ and then sacrificed. Mice in the refeeding group $(n=3)$ were subjected to fasting for $24 \mathrm{~h}$ and then refed with a 50\% (w/w) sucrose/MF diet for further $24 \mathrm{~h}$ and then sacrificed. Mice in the control group ( $n=3$ ) were fed ad libitum with a regular chow diet and sacrificed at the same time as the refeeding group. Total RNA was extracted from the liver and visceral white adipose tissue (WAT).

For the streptozotocin (STZ) study, C57BL/6JJc1 mice (male, 10 weeks) were subjected to fasting for $4 \mathrm{~h}$ before injection with STZ $(n=3)$. STZ $(50 \mathrm{mg} / \mathrm{kg})$ was intraperitoneally injected for $5 \mathrm{~d}$. Control mice were injected with a citrate solution ( $\mathrm{pH} 4.5$ ), which was used as a solvent for preparing STZ solution $(n=3)$. Five days after STZ treatment, plasma glucose levels were evaluated, to confirm the establishment of diabetes (blood glucose level $>250 \mathrm{mg} / \mathrm{dL}$ ).

For direct injection of human insulin, mice were subjected to fasting for a period of $24 \mathrm{~h}$ before treatment. Insulin (Thermo Fisher Scientific, MA, USA) was intraperitoneally injected at a concentration of $8 \mathrm{mU} / \mathrm{g}$, respectively. Mice were sacrificed at specified time points (30 min, $1 \mathrm{~h}, 8 \mathrm{~h}$, and $24 \mathrm{~h}$ ) after injection $(n=3$, at each time point) and the total RNA was extracted from the livers.

\section{$R N A$ extraction and quantitative real-time polymerase chain reaction}

RNA was extracted using TRIzol reagent (Thermo Fisher Scientific, MA, USA) and quantitative polymerase chain reaction (qPCR) was performed using cDNA generated from $1 \mu \mathrm{g}$ of the total RNA with an AffinityScript qPCR cDNA Synthesis kit (Agilent Technologies, CA, USA). The primers used for specified genes were: vaspin: forward, 5'-GAAACATCACAGCCACATTTGT CC-3' and reverse, 5'-CACCCACACATCCACCACTC3'; clebpa: forward, 5'-CAAGAACAGCAACGAGTAC CG-3' and reverse, 5'-GTCACTGGTCAACTCCAGC AC-3'; acidic ribosomal phosphoprotein P0 (36b4): forward, 5'-AAACTGCTGCCTCACATCCG-3' and reverse, 5'-TGGTGCCTCTGGAGATTTTCG-3'. fatty acid synthase (fas): forward, 5'-GGAGGTGGTGATAGCCGG TAT-3' and reverse, 5'-TGGGTAATCCATAGAGCCC

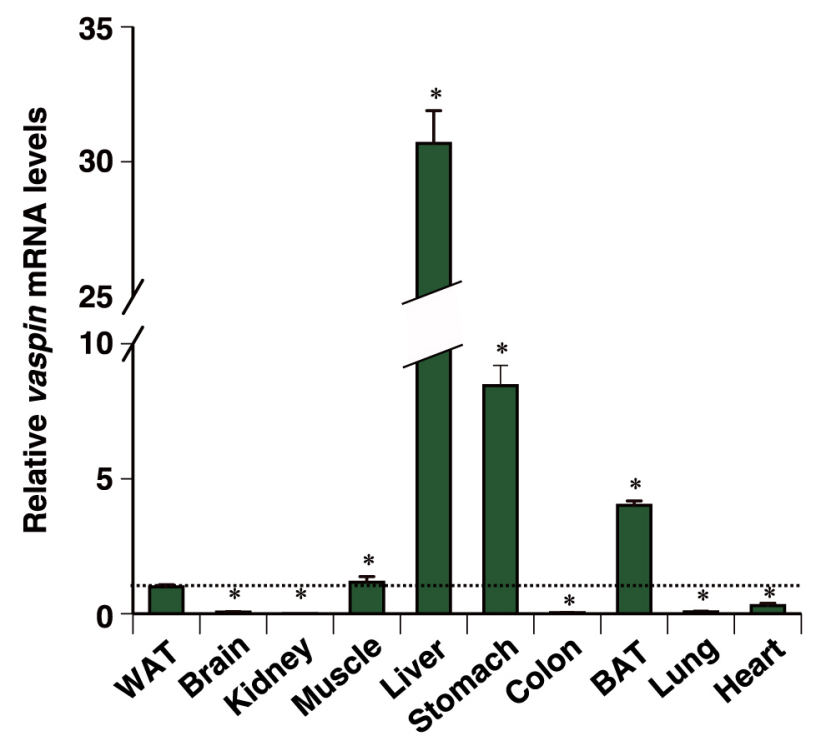

Fig. 1 Tissue distribution of mouse vaspin mRNA. vaspin mRNA levels in WAT, brain, kidney, skeletal muscle, liver, stomach, colon, BAT, lung, and heart tissues from mice were detected by qPCR. Expression levels were normalized to $36 \mathrm{~b} 4 \mathrm{mRNA}$, and each bar represents the average \pm S.E.M. of three individual experiments. WAT, visceral white adipose tissue; BAT, brown adipose tissue. Significant differences from WAT: ${ }^{*} p<0.001$.

AG-3'. Values for sample mRNAs were normalized to expression of $36 \mathrm{~b} 4 \mathrm{mRNA}$.

\section{Statistical analysis}

Quantitative values are presented as mean \pm standard error of the mean (S.E.M.). Differences between mouse groups were confirmed for statistical significance with a two-tailed Student's $t$-test and $p<0.05$ was considered statistically significant.

\section{Results}

\section{Expression of mouse vaspin $m R N A$ is the highest in the liver}

In an earlier report, vaspin mRNA was restricted to visceral white adipose tissue (WAT) in OLETF rats and not detected in the liver [1]. Further, the tissue distribution of mouse vaspin mRNA remains elusive. Thus, we firstly examined vaspin mRNA in each tissue from mice. Although vaspin mRNA was detected in all of the examined tissues, the mRNA levels were the highest in the liver at an approximately 32-fold those in the WAT (Fig. 1).

\section{Fasting-repressed vaspin expression is distinctly induced by refeeding}

It has been reported that fasting and refeeding condi- 
(A) Liver-fas

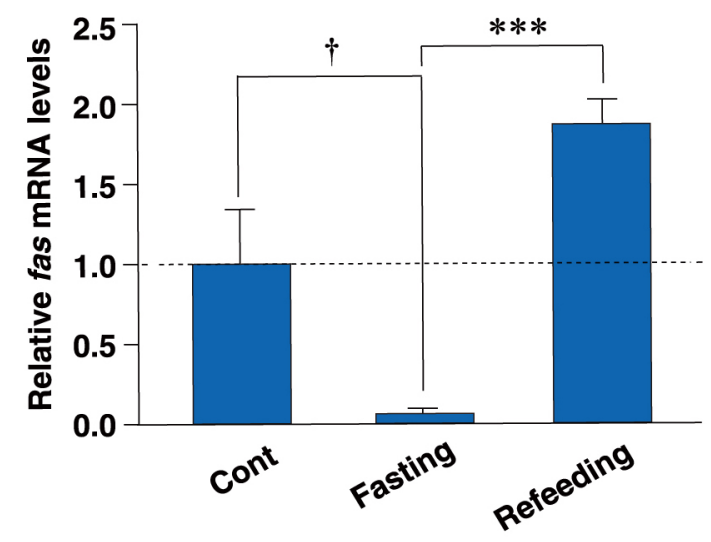

(C) WAT-vaspin

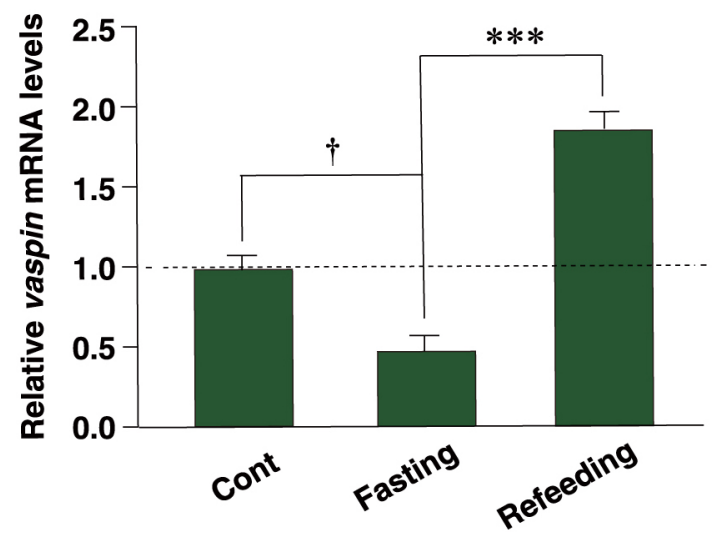

(B) Liver-vaspin

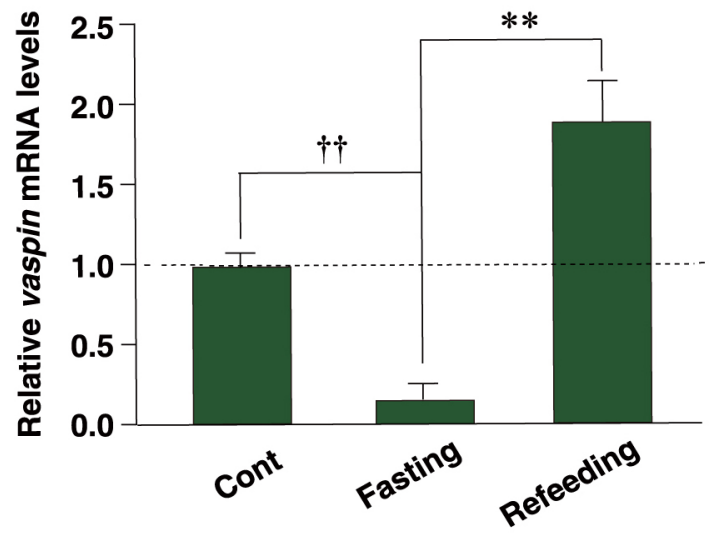

(D) Liver-vaspin

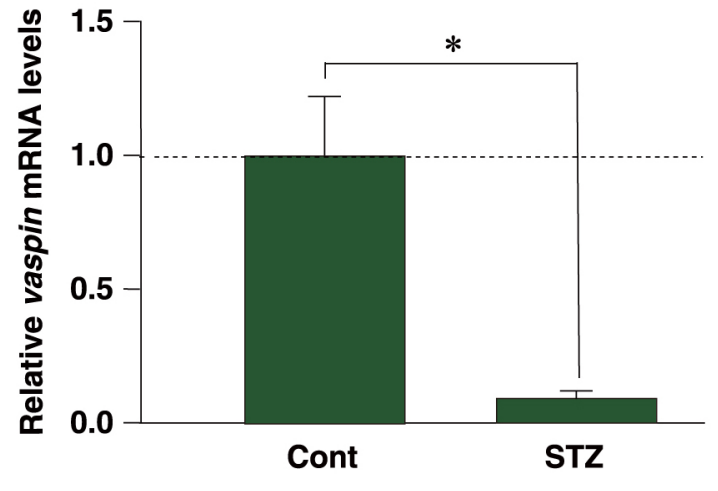

Fig. 2 Refeeding reduced vaspin mRNA expression in the fasting liver.

qPCR analyses of fatty acid synthase (A) and vaspin (B-D) mRNAs were performed using liver (A, B and D) and white adipose (C) tissues from each treated mouse. Expression levels were normalized to $3664 \mathrm{mRNA}$, and each bar represents the average \pm S.E.M. of 3 individual experiments. fas, fatty acid synthase; Cont, ad libitum-fed mice; Fasting, 24 h-fasted mice; Refeeding, mice refed after $24 \mathrm{~h}$ of fasting. STZ, streptozotocin-injected mice. Significant differences compared to Cont: ${ }^{\dagger} p<0.01,{ }^{\dagger \dagger} p<0.001$. Significant differences compared to Fasting: $* p<0.05, * * p<0.01, * * * p<0.001$.

tions potently affect the transcription of several hepatic genes [11]. Therefore, we examined the expression of vaspin under fasting/refeeding conditions in the liver. Fatty acid synthase (fas) mRNA decreased in the fasting liver, but was observed to be induced in the refeeding liver, which was used as a positive control for refeedinginducible genes (Fig. 2A). In the same liver tissue, fasting also markedly caused reduction of hepatic vaspin expression as compared to the control (approximately $10 \%$ of the control). However, vaspin expression was recovered by approximately 2.0 -fold with refeeding, as compared to that of the control (Fig. 2B). Interestingly, vaspin $\mathrm{mRNA}$ in WAT followed a pattern similar to that of hepatic vaspin mRNA (Fig. 2C).

We hypothesized that the increase in vaspin mRNA levels was associated with insulin signals. Further, to examine the potential association between vaspin elevation and insulin, mouse insulin was depleted by destruc- tion of $\beta$ cells by STZ administration. After STZ injection for $5 \mathrm{~d}$, blood glucose levels were found to be above $250 \mathrm{mg} / \mathrm{dL}$ (data not shown). The hepatic vaspin mRNA level was dramatically decreased after STZ injection to approximately $10 \%$ of the control (Fig. 2D). These results suggest that refeeding induces expression of the hepatic vaspin mRNA through the increased insulin levels.

\section{C/EBPa does not have any role in the induction of hepatic vaspin upon refeeding}

To examine whether C/EBP $\alpha$ contributed to the induction of vaspin by refeeding, C/EBP $\alpha$ LKO mice were used in the fasting/refeeding study. The clebpa gene was found to be expressed at markedly lower levels in the fasting or refeeding C/EBP $\alpha$ LKO liver (Fig. 3A). On the other hand, vaspin was induced by approximately 10fold by refeeding in both fasting $\mathrm{C} / \mathrm{EBP} \alpha \mathrm{WT}$ and $\mathrm{C} /$ 

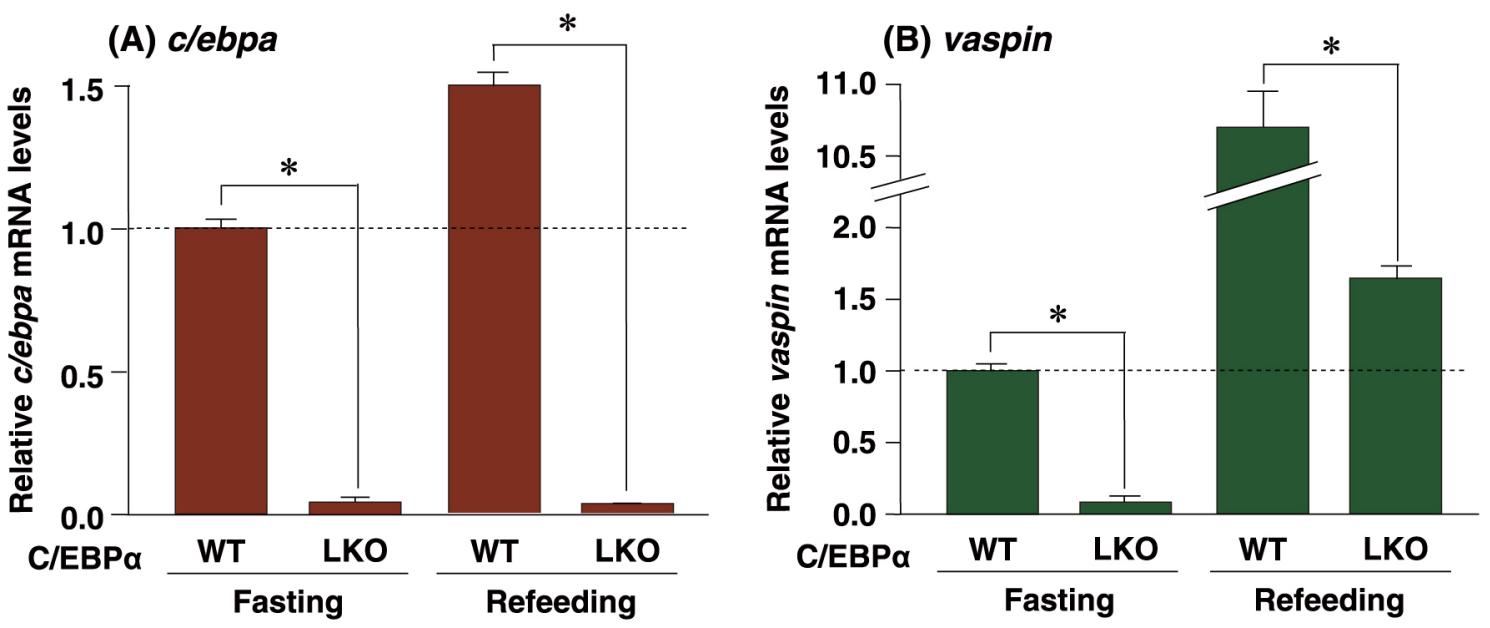

Fig. 3 Effect of deficiency of $\mathrm{C} / \mathrm{EBP} \alpha$ on refeeding-induced hepatic vaspin mRNA.

qPCR analyses of clebpa (A) and vaspin (B) mRNAs were performed using liver samples from each genotyped mouse. Expression levels were normalized to $36 b 4 \mathrm{mRNA}$, and each bar represents the average \pm S.E.M. of 3 individual experiments. WT, C/EBP $\alpha$ wild-type mouse liver; LKO, liver-specific C/EBP $\alpha$ knockout mouse liver. Fasting, mice subjected to 24-h fasting; Refeeding, mice refed after $24 \mathrm{~h}$ of fasting. Significant differences compared with C/EBP $\alpha \mathrm{WT}$ liver: ${ }^{*} p<0.001$.
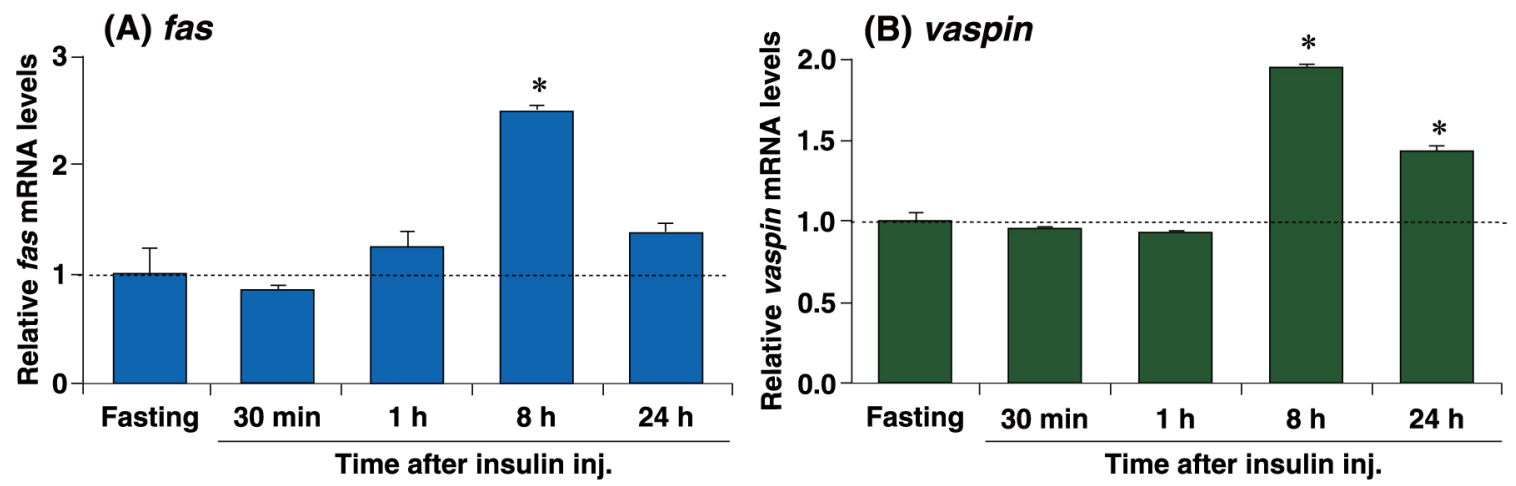

Fig. 4 Hepatic vaspin mRNA expression was induced upon insulin injection.

qPCR analyses of fas (A) and vaspin (B) mRNAs were performed using liver samples from insulin-treated mice. Expression levels were normalized to $3664 \mathrm{mRNA}$, and each bar represents the average \pm S.E.M. of 3 individual experiments. Mice subjected to 24 $\mathrm{h}$ fasting were sacrificed at $30 \mathrm{~min}, 1 \mathrm{~h}, 8 \mathrm{~h}$, and $24 \mathrm{~h}$ after intraperitoneal injection with insulin ( $8 \mathrm{mU} / \mathrm{g}$ of body weight). Significant differences compared to fasting: $* p<0.001$.

EBP $\alpha$ LKO livers (Fig. 3B). These results suggest that C/ $\mathrm{EBP} \alpha$ is not involved in the induction of hepatic vaspin by refeeding.

\section{Insulin induces the expression of fasting-repressed vaspin}

Our results from the refeeding and STZ studies strongly suggested the involvement of insulin in induction of vaspin upon refeeding. To validate this, insulin was directly injected into fasting mice. It has been reported that the expression of fas mRNA is positively regulated by insulin [12]. Insulin was found to significantly induce fas mRNA expression in the fasting liver by approximately 2.5 -fold, $8 \mathrm{~h}$ post injection (Fig. 4A). Furthermore, insulin significantly induced fasting-repressed vaspin mRNA expression by approximately 2.0 and 1.5 fold at $8 \mathrm{~h}$ and $24 \mathrm{~h}$ post injection, respectively (Fig. 4B). These results strongly establish that insulin acts as a positive regulator of hepatic vaspin.

\section{Discussion}

While most of the recent studies on vaspin have focused on its physiological function, only few studies have reported the basis for regulation of the vaspin gene $[13,14]$. Although vaspin mRNA expression of diabetic OLETF rats was restricted to visceral white adipose tissue, mouse vaspin mRNA was highly expressed in the liver. The expression pattern of mouse vaspin mRNA was more similar to that of human vaspin mRNA [4, 5]. 
Furthermore, our result supports the earlier result for mouse vaspin [6]. We have demonstrated that the expression of hepatic vaspin was markedly decreased in liverspecific $\mathrm{C} / \mathrm{EBP} \alpha$ knockout mice. Analysis with a reporter plasmid including the vaspin promoter indicated that two C/EBP-responsive elements (CEBPREs) are necessary for $\mathrm{C} / \mathrm{EBP} \alpha$-dependent induction of vaspin promoter activities. Furthermore, $\mathrm{C} / \mathrm{EBP} \alpha$ in mouse liver was capable of directly binding to the two CEBPREs (Submitted). These results suggested that $\mathrm{C} / \mathrm{EBP} \alpha$ positively regulates hepatic vaspin expression through two functional CEBPREs. The present study revealed that refeeding markedly induced the expression of hepatic vaspin in the fasting liver. Insulin was found to be an important factor for the induction because administered insulin could also induce vaspin expression. Studies on $\mathrm{C} / \mathrm{EBP} \alpha-$ deficient liver indicated that $\mathrm{C} / \mathrm{EBP} \alpha$ is not involved in the induction of hepatic vaspin upon refeeding. Although $\mathrm{C} / \mathrm{EBP} \alpha$ is crucial for basal expression of vaspin in the liver, the induction of vaspin gene by refeeding is likely to be due to other factors, excluding $\mathrm{C} / \mathrm{EBP} \alpha$.

The precise mechanism underlying the induction of vaspin mRNA by insulin or refeeding remains unclear. However, the expression pattern of hepatic vaspin under fasting/refeeding or STZ treatments closely resembles that of some lipogenic genes, such as fas, acetyl CoA carboxylase, and stearoyl CoA desaturase $[12,15]$. The sterol regulatory element binding protein-1c (SREBP-1c) is a master transcriptional regulator of these genes [12, 15]. SREBP-1c is expressed at a low level in the fasting liver, but is dramatically induced upon refeeding, which is mediated by insulin [12]. In addition, SREBP-1c function is activated by insulin at the post-translational level [16]. The activated SREBP-1c homodimer binds to sterol regulatory element (SRE) sequences found on the promoters of its target genes [16]. Further, through the JASPAR database search (http://jaspar.genereg.net/), we found that, typically, three SRE sequences exist within the range of $+1 /-1.5 \mathrm{~kb}$ at vaspin 5 '-upstream: $-508 /-517$ bp, -687/-696 bp, and $-1,037 /-1,046 \mathrm{bp}$. Therefore, hepatic vaspin is likely to be potentially regulated by SREBP-1c.

In summary, the present study demonstrated that the hepatic vaspin gene is positively regulated by insulin. The liver secretes a wide array of hepatokines that exert powerful effects on metabolic processes both in the liver and in the peripheral tissues [17]. Vaspin is able to improve insulin sensitivity and glucose tolerance [17]. Therefore, insulin may have the potential to control insulin sensitivity for peripheral tissues through vaspin secreted from the liver.

\section{Acknowledgments}

This work was supported by a Grant from KAKENHI (grant number 17K08799). We would like to thank Editage (www.editage.jp) for English language editing.

\section{Disclosure}

None of the authors have any potential conflicts of interest associated with this research.

\section{References}

1. Hida K, Wada J, Eguchi J, Zhang H, Baba M, et al. (2005) Visceral adipose tissue-derived serine protease inhibitor: a unique insulin-sensitizing adipocytokine in obesity. Proc Natl Acad Sci U S A 102: 10610-10615.

2. Nakatsuka A, Wada J, Iseda I, Teshigawara S, Higashio K, et al. (2012) Vaspin is an adipokine ameliorating ER stress in obesity as a ligand for cell-surface GRP78/MTJ-1 complex. Diabetes 61: 2823-2832.

3. Nakatsuka A, Wada J, Iseda I, Teshigawara S, Higashio K, et al. (2013) Visceral adipose tissue-derived serine proteinase inhibitor inhibits apoptosis of endothelial cells as a ligand for the cell-surface GRP78/voltage-dependent anion channel complex. Circ Res 112: 771-780.

4. Goktas Z, Owens S, Boylan M, Syn D, Shen CL, et al. (2013) Associations between tissue visfatin/nicotinamide, phosphoribosyltransferase (Nampt), retinol binding protein-4, and vaspin concentrations and insulin resistance in morbidly obese subjects. Mediators Inflamm 2013: 861496
5. Korner A, Neef M, Friebe D, Erbs S, Kratzsch J, et al. (2011) Vaspin is related to gender, puberty and deteriorating insulin sensitivity in children. Int $J$ Obes (Lond) 35: 578-586.

6. Weiner J, Rohde K, Krause K, Zieger K, Kloting N, et al. (2017) Brown adipose tissue (BAT) specific vaspin expression is increased after obesogenic diets and cold exposure and linked to acute changes in DNAmethylation. Mol Metab 6: 482-493.

7. Darlington GJ, Ross SE, MacDougald OA (1998) The role of C/EBP genes in adipocyte differentiation. $\mathrm{J}$ Biol Chem 273: 30057-30060.

8. Ross SE, Erickson RL, Hemati N, MacDougald OA (1999) Glycogen synthase kinase 3 is an insulin-regulated C/EBPalpha kinase. Mol Cell Biol 19: 8433-8441.

9. Inoue $\mathrm{Y}$, Inoue $\mathrm{J}$, Lambert $\mathrm{G}$, Yim SH, Gonzalez FJ (2004) Disruption of hepatic C/EBPalpha results in impaired glucose tolerance and age-dependent hepatosteatosis. J Biol Chem 279: 44740-44748. 
10. Matsusue K, Miyoshi A, Yamano S, Gonzalez FJ (2006) Ligand-activated PPARbeta efficiently represses the induction of LXR-dependent promoter activity through competition with RXR. Mol Cell Endocrinol 256: 23-33.

11. Altarejos JY, Montminy M (2011) CREB and the CRTC co-activators: sensors for hormonal and metabolic signals. Nat Rev Mol Cell Biol 12: 141-151.

12. Shimomura I, Bashmakov Y, Ikemoto S, Horton JD, Brown MS, et al. (1999) Insulin selectively increases SREBP-1c mRNA in the livers of rats with streptozotocininduced diabetes. Proc Natl Acad Sci U S A 96: 1365613661.

13. Marra F, Bertolani C (2009) Adipokines in liver diseases. Hepatology 50: 957-969.

14. Escote X, Gomez-Zorita S, Lopez-Yoldi M, Milton-
Laskibar I, Fernandez-Quintela A, et al. (2017) Role of omentin, vaspin, cardiotrophin-1, TWEAK and NOV/ CCN3 in obesity and diabetes development. Int J Mol Sci 18: 1770 .

15. Shimano H, Yahagi N, Amemiya-Kudo M, Hasty AH, Osuga J, et al. (1999) Sterol regulatory element-binding protein-1 as a key transcription factor for nutritional induction of lipogenic enzyme genes. J Biol Chem 274: 35832-35839.

16. Xu X, So JS, Park JG, Lee AH (2013) Transcriptional control of hepatic lipid metabolism by SREBP and ChREBP. Semin Liver Dis 33: 301-311.

17. Watt MJ, Miotto PM, De Nardo W, Montgomery MK (2019) The liver as an endocrine organ-linking NAFLD and insulin resistance. Endocr Rev in press. 